\title{
La langue française dans la presse du Burkina Faso
}

Nataša Raschi Faculté de Lettres et Philosophie de l’Université de Pérouse (Italie)

Petit pays fait de terre, l'ancienne colonie française de la Haute-Volta change définitivement son nom en Burkina Faso le 4 août 1984. Il s'agit toujours d'un nom composé et il est toujours question de pays (Somé 109). Si l'ancien nom était lié aux trois plus grands fleuves qui traversent le territoire, à savoir Volta noire, Volta blanche et Volta rouge, la nouvelle appellation signifie « le pays des Hommes intègres » et est composée de deux substantifs : le mooré Burkina ou « homme intègre », le jula Faso ou « terre, pays ». De même, l'adjectif de nationalité burkinabè, se construit en ajoutant à cette même racine mooré le suffixe bè, qui en fulfulde (ou peul) signifie « les gens de ». Dès sa formation, l'adjectif burkinabè a été déclaré invariable en genre et en nombre.

Les frontières du Burkina Faso touchent le Mali au Nord, le Niger à l'Est et le Bénin, le Togo, le Nigéria et la Côte d'Ivoire au Sud. La route principale qui relie l'ancienne capitale Bobo-Dioulasso ou "maison des Bobo-Dioula", à Gorom-Gorom ou "pays des tentes bleues", ville du fabuleux marché du Sahel, traverse le pays du Sud au Nord, laissant le visiteur stupéfait de la variété des paysages et des gens. Alors que le Sud est vert, riche en produits agricoles et en attractions touristiques, le Nord présente toutes les caractéristiques des régions typiquement sahéliennes, avec un paysage de steppes brûlées par la sécheresse, où affleurent quelques grandes dunes qui annoncent l'approche du désert. Bien que l'exode rural y sévisse - comme dans toute l'Afrique Noire - et qu'une grande métropole comme Ouagadougou s'y développe, le Burkina Faso est resté un pays de villages où le trait dominant est partout déterminé par la chaleur de l'accueil et de l'hospitalité.

Le pays présente beaucoup d'originalité sur les plans géographique et historique, d'où résulte une richesse culturelle dont le capital initial est vieux de plusieurs millénaires. C'est surtout l'idée de ce pays en tant que " carrefour des peuples » (Da 6-7) qui se révèle particulièrement vraie en raison de sa situation géographique.

La population du Burkina Faso est composée d'une soixantaine d'ethnies d'importance numérique inégale qui n'occupent pas toujours des aires géographiques bien précises. Comme dans la plupart des pays africains, les frontières ethniques à l'intérieur du pays ne sont pas toujours nettes et les multiples groupes n'ont été tenus en aucune considération à l'époque de la disposition des confins de la part des puissances coloniales. On ne peut en outre oublier les raisons contingentes, c'est-à-dire la grande mobilité des 
Moosé, des Dioula, des Peul, à la recherche de terres fertiles ou de pâturages et en raison de leurs activités commerciales.

Toutes les ethnies du pays peuvent être regroupées en une dizaine de familles :

- sur le plateau central du pays, les Mossi dont l'ethnie est la plus importante, vu qu'elle compterait quelque cinq millions d'habitants sur « une population d'environ 12 millions d'habitants » (Somé 109) ;

- à l'est, les Gulmanceba ;

- au sud, les Gurunsi et leurs voisins les Bissa ;

- le nord est partagé entre les Peul, les Touareg, les Bella, les Dogon ;

- à l'Ouest, où l'éclatement ethnique est plus important, on distingue les groupes Bobo et Bwa, le groupe Senoufo, le groupe Lobi et beaucoup d'autres aussi (Da 7-8).

Ce qui rend la description encore plus variée et multiforme est le fait que, à l'intérieur de ces groupes, d'autres sont venus s'insérer. Certains, qui se sont spécialisés dans le commerce (dont la langue incontestée est le jula), sont disséminés dans tout le pays, tandis que quelques peuples du Sahel (Peul et Touareg surtout) sont restés nomades. Parmi les peuples les plus anciennement installés figurent les Bobo et les Sénoufo. Il existe des populations Mandé venues de la haute vallée du Niger, ainsi que des populations néosoudaniennes qui dans le passé ont formé de puissants royaumes.

Il en découle que le morcellement est considérable et que chaque ethnie possède sa langue ou son dialecte. En particulier, « trois langues ${ }^{1}$ ont été retenues comme nationales : le Moore de l'ethnie majoritaire, le Jula des commerçants et le Fulfuldé des éleveurs » (Da 8) et cela en raison de leur extension géographique. À l'image de beaucoup d'autres pays africains, le Burkina Faso est donc un pays multiethnique et multilingue. Selon le statut, les fonctions et les rapports de force qui caractérisent les diverses langues, on peut encore distinguer les langues nationales d'un côté et le français, en tant que langue officielle, de l'autre. Distinction établie par l'article 35 de la Constitution du 27 janvier 1997: «La langue officielle est le français. Une loi fixe les modalités de promotion et d'officialisation des langues nationales. » (Nikiema 16). Ce qui souligne le fait qu'encore aujourd'hui, on assiste à un grand décalage entre l'enseignement en langue française - dans ce cas seulement on parle de scolarisation - et l'enseignement en langues nationales - pour lequel on utilise le terme alphabétisation².

La langue française occupe également la totalité des journaux burkinabè. Notre corpus - repéré du $1^{\text {er }}$ septembre 2006 au $1^{\text {er }}$ mars 2007 - a été téléchargé du site

\footnotetext{
${ }^{1}$ A ce propos, Pascal Somé souligne le fait important qu'il ne s'agit pas de «simples dialectes mais de langues à part entière » (Some 109).

${ }^{2}$ Quel en est le résultat ? Un pays où seulement $20 \%$ de la population a la possibilité d'arriver à maîtriser la langue française aussi bien à l'oral qu'à l'écrit (Some 111).
} 
www.lefaso.net qui offre toute l'actualité du pays en ligne ${ }^{3}$. Notre objectif est celui de repérer et d'analyser les phénomènes lexicaux et morphosyntaxiques les plus récurrents pour arriver à évaluer la portée de la langue de l'Hexagone, dans un contexte où elle se trouve nécessairement à coexister avec d'autres langues ethniques. Pour ce qui est de la méthodologie, nous aborderons ces variantes sur la base d'une lecture contrastive et avec une attention particulière aux mécanismes de formation des expressions les plus intéressantes. Le sujet abordé est vaste et seuls deux articles lui ont été consacrés (Gandon, Keita). Certaines catégories de spécificités du français donnent ici l'impression qu'il s'agit d'erreurs ou de maladresses de la part des journalistes mentionnés, alors que d'autres parties démontrent un souci de la langue et une volonté de créer des effets précis sur les lecteurs. Nous devons alors préciser que tout cela est dû à notre volonté de tracer les nervures principales de la problématique annoncée pour en déterminer un bilan assez complet.

Parmi les quotidiens figurent Sidwaya, journal d'Etat dont le titre en mooré signifie la vérité est arrivée, le Pays, un autre quotidien qui se veut apolitique et l'Observateur Paalga - ce dernier terme en langue mooré signifie « Nouveau »-, quotidien qui se veut indépendant, le plus lu et le plus ancien, fermé pendant la révolution et puis ouvert à nouveau. Tous les vendredis y paraît la rubrique Une lettre pour Laye construite sur l'exemple des Lettres persanes. En fait, Laye est un petit village situé à une vingtaine de kilomètres de Ouagadougou, sur l'axe routier Ouagadougou-Ouahigouya ; il symbolise le village éloigné où les tumultes de la ville arrivent difficilement. La lettre hebdomadaire de Passék Taalé - en mooré «ça ne vaut pas la peine qu'on s'en veuille les uns les autres, ça ne vaut pas la peine qu'on fasse une bagarre pour si peu » - apporte donc des nouvelles des turbulences de la ville au village. Les longues missives sont adressées au cousin Wambi, prénom donné aux nouveaux-nés (sans distinction de sexe) et signifiant "petit masque » de Wango = masque et bila= petit ${ }^{4}$. Pour ce qui est des hebdomadaires, nous trouvons Bendré, qui en mooré signifie tambour-gourde ${ }^{5}$, donc tambour et tambourinaire qui donne et élargit les informations, le Journal du Jeudi, un satirique qui sort le jeudi et qui contient une rubrique particulièrement appréciée Moi, Goama (ou Gouama en moore: paroles, discussions) (Sissao 5) où l'on trouve le commentaire d'un fait politique en « petit nègre» sur le modèle de la Chronique de Moussa d'Ivoire-Dimanche en Côte d'Ivoire

\footnotetext{
3 Nous sommes reconnaissants à Monsieur Michel SAWADOGO, Deuxième Conseiller auprès de l'Ambassade du BURKINA FASO à Rome, pour toutes les informations qu'il nous a données et pour son aide ponctuelle.

${ }^{4}$ Habituellement ce prénom est donné à un enfant, parce qu'on veut être reconnaissant envers un masque auprès duquel, une femme connaissant des problèmes de maternité, est allée confier ses soucis et a obtenu gain de cause.

${ }_{5}$ On ne peut pas ne pas rappeler à ce propos la bendrologie (Cf. ILBOUDO, Patrick G. [propos recueillis par], « Frédéric Titinga Pacéré : Mon testament littéraire », in Notre Librairie, n 101, avril-juin 1990, p. 65-66), ou étude du langage des tam-tam, ainsi que l'immense œuvre de Maître Frédéric Titinga Pacéré, dont deux titres particulièrement importants sont: Bendrologie et littérature culturelle (1987) et Bendr N'Gombe : Parole et poésie du tam-tam (1988).
} 
(Gandon 87). Il existe aussi L'Hebdomadaire, proche du gouvernement, l'Indépendant, un autre hebdomadaire qui se veut d'investigation, dont le fondateur Norbert Zongo est un journaliste très célèbre assassiné le 13 décembre 1998 à Sapouy. Suivent un hebdomadaire au titre jula Sanfinna - mot dioula composé de «San» (pluie) et «finna » (noir); sa traduction pourrait être le ciel est noir, les temps sont troubles, il y a une menace, un danger - et L'Opinion. Encore, l'Evénement est un bimensuel et se veut journal d'investigation.

\section{Considérations lexicales}

Après la définition de notre corpus, nous avons relevé toutes les particularités lexicales qui semblaient présenter un caractère d'étrangeté. Certains éléments ont été exclus après vérification de leur existence en français de base (la norme étant à ce stade représentée par Le Petit Robert). Ceux qui sont restés pour la seconde phase ont été cherchés dans le Grand Robert' ainsi que dans l'Inventaire des particularités lexicales du Français en Afrique noire, ouvrage de référence en la matière, dont le numéro 6 est entièrement consacré à la langue française du Burkina Faso ${ }^{7}$. Nous allons donc concentrer notre étude sur les particularités lexematiques des unités non attestées en français ou des néologismes de forme.

Les tendances les plus évidentes sont déterminées par les emprunts (à une langue locale, à une langue africaine non locale, à une langue non africaine). À cela, il faut ajouter l'inexistence de certaines réalités africaines dans l'expérience française, aspect non secondaire vu que l'existence d'un mot implique celle d'un référent imaginaire ou concret. On assiste ainsi à l'apparition d'une unité nouvelle, il s'agit d'un mot africain intégré dans la langue et dont le comportement ne semble pas se distinguer des autres parce qu'il suit les règles morphologiques de la langue $\mathrm{d}^{\prime}$ accueil ${ }^{8}$. Un exemple très répandu est représenté par le mot baobab, le gros arbre des savanes considéré comme un fétiche, ou comme l'arbre de la vie ; régulièrement inséré dans Le Petit Robert, il assume également la marque du pluriel. Dans notre corpus, on peut lire Un baobab est tombé (Karama, A. K. «Décès de Joseph Ki-Zerbo », Sidwaya, 06/12/2006) lors du décès de Ki-Zerbo, le grand historien burkinabè, auteur de la première et (dans son cas) monumentale Histoire d'Afrique, avec l'utilisation de ce terme au sens métaphorique pour désigner un homme d'un statut extraordinaire, grand dinosaure de l'histoire culturelle et politique non seulement de son pays, mais aussi de tout le continent ${ }^{9}$.

\footnotetext{
${ }^{6}$ Il s'agit du dictionnaire suivant: Le Grand Robert de la langue française : dictionnaire alphabétique et analogique de la langue française, Paris, Le Robert, 1985, 2ème édition revue et enrichie par Alain Rey.

${ }_{7}$ Consulter à ce propos le site www.unice.fr/ILF-CNRS/ofcaf/6 plus l'indicatif de la lettre de l'alphabet suivi de.pdf

8 Comme le dit Pierre Dumont: «La plupart des emprunts sont en cours de francisation phonologique, graphique, morphosyntaxique et même sémantique [...] » (Dumont 145).

${ }^{9} \mathrm{~A}$ ce propos, on se souvient du fait que le français de France utilise le parallèle avec le chêne pour décrire une situation pareille, par exemple : « ces chênes qu'on abat ».
} 
L'occasion d'une nouvelle de ce genre ouvre la voie à toute une série d'articles très importants pour notre recherche parce qu'ils empruntent plusieurs termes aux langues locales et surtout à la culture ethnique. On commence par « les vieux mossé bloquent le cortège afin d'offrir le sacrifice de deux crapauds à la mémoire du vieux samo Ki-Zerbo » (Ouedraogo, D. E. "Joseph Ki-Zerbo conduit à sa dernière demeure », Sidwaya, 09/12/2006). Les deux termes soulignés indiquent deux ethnies du pays, c'est-à-dire les Mossé - plus souvent Mossi - qui sont les plus nombreux et les Samo qui constituent une des populations venues de la haute vallée du Niger qui ont occupé les marges du pays mossi. Mossé, est le pluriel de l'ethnonyme en langue mooré, au singulier moaga et samo désigne l'ethnie du défunt. Cela dénote l'importance de cet homme pour tous les habitants du pays et donc sa valeur qui dépasse l'appartenance ethnique.

À partir des «[...] lieux sacrés du Moogho» (Taalé, P. "Quand les dieux se rencontrent ", Observateur Paalga, 15/12/06), on arrive au "communiqué "moronabal" [qui] enjoignait aux Mossis des villes que le cortège funèbre traverserait d'exiger et de prélever les droits coutumiers d'usage sous peine de mise en fourrière de la dépouille du grand Samogho » (Taalé, P. «Le gnontoro va couler à gogo », Observateur Paalga, 08/12/06). Tous les rituels des funérailles doivent être respectés pour la gloire du personnage et le lexique suit nécessairement la tradition. L'interprétation est parfois incertaine à cause des variantes graphiques. En effet, la graphie du premier nom souligné, d'origine mooré, n'est pas fixée. On trouve mogho, morho, mogo, moro aussi. Il s'agit du titre porté par le souverain de l'empire mossi, vivant à Ouagadougou et considéré comme le suzerain de tous les autres chefs mossi ${ }^{10}$. La chefferie traditionnelle est encore puissante au Burkina et elle existe parallèlement à la vie politique moderne. Les chefs des quatre royaumes mossi sont: le morho-naba de Ouagadougou, le Yatenga-naba, le Tenkodogo-naba et le Fada N'Gourma naba. L'adjectif «moronabal» est une formation locale venant du nom "morho-naba » et se compose d'une racine ethnique à laquelle on a ajouté un suffixe français. Il faut encore préciser que seulement dans ce cas, nous avons trouvé le nom Mossi accordé au pluriel comme s'il s'agissait d'un nom français.

Un autre exemple de dérivation régulière, au féminin cette fois-ci, se trouve dans le passage suivant qui continue la description des funérailles : «Dans les rues, les dolotières sont présentes. » (Ouedraogo, D. E. «...le jour de l'inhumation », Sidwaya, 09/12/2006). La presse burkinabè retranscrit ce même terme à plusieurs reprises et nous en donne la racine : «Le dolo, la célèbre bière de Sorgho rouge » ou de mil appelée également « gnontoro » (Taalé, P. « Le gnontoro va couler à gogo », Observateur Paalga, 08/12/06) terme $\mathrm{du}$ dialecte samo (ou san) équivalent au dolo ou raam des mossé. Il s'agit donc d'un nom d'origine jula auquel on ajoute le suffixe français pour désigner les femmes qui confectionnent et vendent sur le marché ce type de bière. Comme le souligne Gisèle

${ }^{10}$ D'après : www.unice.fr/ILF-CNRS/ofcaf/6/M.pdf 
Prignitz, ce sont surtout «les réalias [qui] émaillent le discours sous forme de particularités lexicales » (Prignitz 6).

À propos de l'application des règles morphologiques pour les emprunts, l'exemple suivant est doublement intéressant: "C'est exactement à $14 \mathrm{~h} 47 \mathrm{mn}$ que le cercueil de Joseph Ki-Zerbo a été mis dans le caveau au son de tam-tams, de coups de fusil et des youyou des femmes Samo» (Ouedraogo, D. E. «...le jour de l'inhumation », Sidwaya, 09/12/2006). L'on remarque que le premier mot souligné, régulièrement inséré dans Le Petit Robert, présente la marque du pluriel, tandis que le second en est dépourvu et désigne ici les cris traditionnels de joie ou de colère. Ce dernier existe aussi dans le dictionnaire français, mais seulement dans le langage de la marine.

L'utilisation des emprunts peut intervenir pour augmenter le niveau d'attention, ainsi que pour souligner l'appartenance à une aire géographique et donc positionner immédiatement l'attention du lecteur sur un endroit bien précis du pays. Lorsque l'on retranscrit un refrain particulièrement cher à Ki-Zerbo : "Nan an laara an saara » (Karama, K. A. «VIII ${ }^{e}$ anniversaire du drame de Sapouy », Sidwaya, 14/12/2006) expliqué plus tard dans le même article par les mots suivants "Maintenant, la parole est à l'action... ", on souligne son appartenance ethnique.

Dans la plupart des articles pris en considération, les mots ethniques sont presque toujours accompagnés de la traduction en langue française, pour en faciliter la compréhension même en dehors des frontières nationales, vu la publication sur la Toile de la totalité des articles de la presse burkinabè. Les différentes modalités traductives sont représentées par les exemples qui suivent :

a. «Il y a le développement des infrastructures à l'image des marchés à bétail ou yaars » (Coulibaly, S. N. "Faire de Koudougou, Ouahigouya et Fada N'Gourma des pôles régionaux de développement ", Sidwaya, 16/12/2006), le mot en langue ethnique accompagne son correspondant en langue française en occupant la même position, donc en ayant la même valeur ;

b. «[...] un péyô (petit marché) se tiendra le samedi [...]» (Taalé, P. «Quand les dieux se rencontrent », Observateur Paalga, 15/12/06), ici la traduction en français se trouve à l'écart parce que placée entre parenthèses ;

c. on trouve également une autre modalité explicative : pour un cas de vandalisme, on cite l'école «Waog taaba», nom suivi de sa traduction « Les vandales savent-ils seulement que “Waog-taaba", en langue mooré, véhicule une invite au respect mutuel ?» (Taalé, P. «L'offre de Gbagbo », Observateur Paalga, 11/09/06).

La dérivation est un procédé aussi productif que l'emprunt et elle s'effectue en suivant un système tout à fait régulier pour le français, mais elle a pour conséquence de créer de nouvelles unités lexicales. 
Le préfixe in- assume une connotation négative de critique ouverte et ironique visà-vis de ce commerce dit «équitable» par les pays occidentaux et considéré comme inacceptable de la part des pays africains : "Le SIAO 2006 est une occasion de crier haro sur le commerce inéquitable » (JJ, «Artisanat. L'extérieur fait son marché au détriment de la consommation locale », Journal $d u$ Jeudi, $\left.\mathrm{n}^{\circ} 789,02-08 / 11 / 06\right)$. Cette même valeur oppositive se reproduit par exemple dans le titre «Le discours “indiscourable" d'Ali Traoré » (Taalé, P. «Le discours "indiscourable" d'Ali Traoré », Observateur Paalga, 19/01/07), dans le cas d'un général qui devait prononcer une allocution lors d'une cérémonie officielle et qui par contre en a été empêché, question de succession au pouvoir, donc d'exclusion.

Les situations d'urgence où verse l'actualité ivoirienne touchent de près le Burkina, vu que bon nombre d'émigrés-travailleurs ont été rapidement rapatriés après l'explosion du conflit. À ce propos, le Président burkinabè a été l'un des plus actifs dans la recherche d'une solution durable, ce qui depuis le début impose certains choix d'ordre pragmatique aux journalistes. Considérons à cet égard la phrase suivante : «Le thème principal sera le dialogue interivoirien » (Ibidem). Double est l'intérêt de cet adjectif : in- renvoie à l'intérieur de la Côte d'Ivoire et son composé inter- amplifie la portée du discours à la totalité des ethnies du pays.

Même procédé pour la suffixation. Les néologismes nés par suffixation font toujours appel à des suffixes encore productifs en français, principalement -iste. L'originalité de ce phénomène tient donc moins aux procédés utilisés qu'aux éléments qui en sont affectés. On les retrouve fort classiquement sur une base constituée par un nom propre. L'adjectif que l'on lit le plus souvent dans la presse burkinabè est « sankariste » du nom de l'ancien Président Thomas Sankara assassiné le 15 octobre 1987, date qui marquera profondément l'histoire du pays. Depuis cet événement, les «partis politiques et les associations sankaristes » organisent un peu partout nombre de manifestations commémoratives.

Un autre système de dérivation par suffixation consiste dans le fait de copier des formules typiques de la langue anglaise. Avant tout, l'expression née de la formation du génitif saxon «Simonville » (Taalé, P. «L'offre de Gbagbo », Observateur Paalga, 11/09/06), ou Ouagadougou, dont le maire est Simon Compaoré, pour souligner le sentiment de la propriété et cacher - par l'effet de mise à distance - la critique adressée à la famille du Président de la République, qui gère tous les postes du pouvoir dans le pays. Ensuite, une autre formule qui relève du génitif et qui sert à mettre en évidence un métier particulièrement important dans l'économie du pays. Il s'agit des cotonculteurs (Rédaction Sidwaya, "Ouédraogo Fadoul Georges, PDG du groupe Fadoul Afrique », Sidwaya, 04/12/2006), là où le coton est appelé « notre or blanc» (JJ, « Relations internationales. Les Burkinabè commercent avec la Chine quand leurs leaders dissertent avec Taïwan », Journal du Jeudi, $\mathrm{n}^{\circ}$ 792, 23-29/11/06) en raison de l'importance de cette exportation vers les pays de l'Extrême-Orient. Gisèle Prignitz explique les emprunts à l'anglais comme déterminé par 
un goût aussi bien de modernité que de snobisme (Prignitz 57). Mais il est d'autant plus vrai que la communication au niveau mondial est véhiculée par l'anglais et que plusieurs pays limitrophes sont anglophones, deux éléments qui ne sont probablement pas secondaires pour les journalistes du Burkina Faso.

Le même effet est obtenu par la retranscription insistée de deux adjectifs à valeur nettement opposée comme dans l'exemple : «Le congrès d'un grand parti n'est pas petit. Le Congrès pour la démocratie et le progrès (CDP) est en congrès. [...] ce grand rassemblement des grands » (JJ, «CDP. Le congrès d'un grand parti n'est pas petit », Journal du Jeudi, $\mathrm{n}^{\circ}$ 790, 09-15/11/06), mais encore plus intéressant est le fait de disposer ces mêmes adjectifs comme s'il s'agissait d'un chiasme : "Un grand n'est pas petit, donc ses petits défauts sont forcément grands ».

À propos de répétition, dans maints articles l'on remarque la tendance à abuser de l'adverbe «bien». Ce dernier intervient très fréquemment dans un sens de critique ouverte à la politique en acte, qui semble exagérer avec les décorations le jour de la Fête de la République le 11 décembre s'il est vrai que : «Ce fut d'ailleurs pendant longtemps une aberration, car s'il y a bien des gens qui s'échinent jour et nuit dans des domaines concrets de création de richesses, c'est bien dans le secteur du privé. Donc la IVe République a bien démocratisé la reconnaissance des efforts des uns et des autres. » (JJ, « 11 Décembre. Vive la République et vive les honneurs!", Journal du Jeudi, $\mathrm{n}^{\circ}$ 795, 14-20/12/06). Même en français standard, cet adverbe sert à créer l'insistance, mais ici la répétition est voulue et l'effet recherché est justement l'amplification de la critique.

Un système typique de la presse française consiste dans l'insertion d'acronymes. Dans le cas de la presse burkinabè, il s'agit de sigles toujours très faciles à décrypter parce tous accompagnés par la formule en entier. Cela indique que probablement ces articles sont lus surtout à l'extérieur du pays. On trouve par exemple : "Parti de la renaissance nationale $(P A R E N),[\ldots]$ Parti pour la convergence et le progrès $(P C P),[\ldots]$ Convention des forces républicaines $(C F R)$ » (Taalé, $P$. «Les bouchers font la grève du couteau», Observateur Paalga, 24/11/06). Les deux seuls sigles qui ne présentent aucune explication sont FESPACO (Festival Panafricain du Cinéma et de la Télévision de Ouagadougou) et SIAO (Salon International de l'Artisanat de Ouagadougou) parce qu'ils existent depuis longtemps dans le pays et en constituent une vitrine exceptionnelle par rapport à la totalité du continent africain.

Un phénomène particulier est lié à l'utilisation d'expressions figées en français, mais remodulées sur un aspect très spécifique de la réalité africaine. Ainsi, peut-on avoir des noms qui se comportent de cette façon ou des expressions tout entières. Par exemple, « À la récréation, ceux qui ne boivent pas la bière prennent de la sucrerie » (Ouedraogo, D. E. «Débits de boissons à proximité des établissements scolaires », Sidwaya, 15/12/2006). Ce terme désigne ici une boisson rafraîchissante sucrée mais non alcoolisée, genre Coca, Fanta 
et autres. Dans un autre article, l'on remarque l'expression bien connue de campagnes de masse (Ouedraogo, D. E. "Lutte contre la bilharziose dans la Boucle du Mouhoun », Sidwaya, 13/12/2006) lorsqu'un laboratoire pharmaceutique distribue ses comprimés à toute la population du pays, même si ceux qui doivent vérifier ne maîtrisent pas la posologie à administrer. Cette information laisse entrevoir par sa généralisation une nuance d'amertume envers un projet de toute évidence exclusivement politique et ne manque pas d'évoquer de bien tristes épisodes qui se vérifient au quotidien sur tout le continent africain ${ }^{11}$.

La réalité sociale burkinabè est liée à l'expression parenté à plaisanterie (autres synonymes rakiré ou dakiré en mooré et plaisanterie parentale, traduction d'une expression

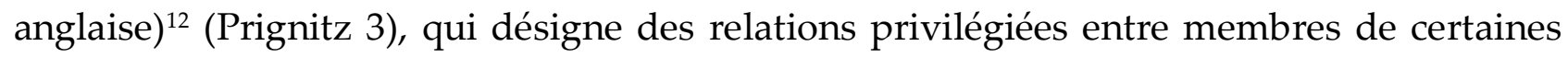
familles, classes d'âge et ethnies différentes qui permettent d'entretenir dans la conversation un ton très détendu, critique et agréable ${ }^{13}$. Ses origines sont souvent liées à des événements historiques communs, mais parfois aussi à quelques faits divers anecdotiques. L'intérêt de cette relation réside dans l'interaction des personnes ou des groupes concernés ${ }^{14}$.

Les phénomènes lexicaux jusqu'ici envisagés touchent à l'identité des mots, mais l'une des caractéristiques majeures de la modification du lexique français en Afrique tient à la manière dont ces mots se combinent avec leur contexte. On a parfois le sentiment que le langage journalistique en arrive à imposer certaines locutions qui ne constituent pourtant pas un mot de passe valable sous tous les cieux, parce qu'elles sont retravaillées sur la base de la situation locale contingente. Lorsque l'actualité est occupée par un sujet particulier, toute une panoplie de néologismes de différente nature surgit. Paradoxalement, il semble que la notion de familiarité se reconstruise différemment de ce qui se passe en français de base. En effet, le phénomène de mise à distance semble souvent extrêmement lié à la présence d'une expression imagée, totalement figée dans le contexte originaire, au point que les locuteurs français n'y lisent plus que son sens littéral. Moins imprégnés de l'usage, nos locuteurs africains, tout en accréditant le sens figuré de l'expression, semblent se désolidariser de la hardiesse de l'image pour en souligner le sens figuré. Leur insertion dans ces articles produit toujours un effet de mise en relief et donc ne fait qu'augmenter l'attention du lecteur. Les expressions qui suivent sont intéressantes parce qu'elles naissent de l'actualité locale. À leur égard, il est impossible de parler $d^{\prime}$ africanismes parce qu'elles n'apparaissent qu'une seule fois dans notre corpus. Il s'agit

\footnotetext{
${ }_{11}$ Voir à ce propos le film de Fernando Meirelles, La Constance du jardinier de 2005, tiré du roman de John le Carré de 2001 ayant le même titre.

12 Tous ces synonymes sont présents dans l'article suivant : OUEDRAOGO, Daouda Emile « Joseph Ki-Zerbo conduit à sa dernière demeure », Sidwaya, 09/12/2006.

${ }^{13}$ D'après : www.unice.fr/ILF-CNRS/ofcaf/6/P.pdf

${ }^{14}$ Les Samo sont les parents à plaisanterie des Mossé (Klotchkoff 55).
} 
plutôt de calques engendrés sur la base d'une extension d'emplois et de sens, parce que liés à l'urgence du Congrès annuel du Parti du Président (Dumont 141-158).

Ainsi, trouve-t-on un néologisme composé de deux substantifs qui désignent l'identification des deux éléments et une correspondance parfaite comme dans l'exemple qui suit: «le parti-pouvoir comme le CDP» (Rédaction Sidwaya, "Ouédraogo Fadoul Georges, PDG du groupe Fadoul Afrique », Sidwaya, 04/12/2006). Il est bien de remarquer qu'en français de base la préposition au centre de cette expression est fondamentale dans le sens que l'on parle du parti au pouvoir dans un moment bien précis, tandis qu'en Afrique l'on a vécu et souvent l'on vit encore dans des réalités ayant un parti unique qui incarne la totalité du pouvoir.

Une autre expression très intéressante trouve son modèle dans le français standard «État-providence » pour être ensuite remodulée sur la réalité du pays : «Qui paie gagne : le soleil des partis-providence décline. » (JJ, «Législatives 2007. Qui paie gagne : le soleil des partis-providence décline », Journal du Jeudi, $\mathrm{n}^{\circ}$ 800, 18-24/01/07). Ici les deux noms, dont l'un variable et l'autre invariable, restent liés par un trait d'union, ce qui dénote la puissance, économique surtout, d'un système politique dominé par l'argent.

Sur l'exemple du français standard «bonification d'ancienneté », un néologisme né de la couleur locale se trouve à la fin d'un article qui commémore le jour de la République le 11 décembre 2006. La critique ne manque pas d'ajouter que les décorés « se ruent dès le lendemain sur les fameux papiers ministres pour formuler leur demande de bonification d'échelon avec le j'ai l'honneur de circonstance!» (JJ, «11 Décembre. Vive la République et vive les honneurs!", Journal $d u$ Jeudi, $\left.\mathrm{n}^{\circ} 795,14-20 / 12 / 06\right)$, ce qui signifie qu'on veut toujours monter en grade et surtout qu'on veut le faire de manière très égoïste, en jouant au chacun pour soi, et très fausse, en répétant toujours les mêmes formules de circonstance.

La formule qui utilise le pronom personnel « je» plus le verbe correspondant est très répandue, surtout lorsqu'il s'agit d'expressions concernant le monde de l'argent. Dans tous ces cas, on assiste alors à une extension de sens. Quand on compare par exemple les «Burkinabè façon » aux «Voltaïques, eux, étaient modestes!» (JJ, « Social. Les Burkinabè façon du Burkina Faso », Journal du Jeudi, n 796-797, 21/12-03/01/07) et quand on souligne également qu'ils pratiquent les «je retiens » ou soupent avec « la sauce au gombo ». Le gombo est une plante typique dont le fruit sert de condiment et pour la composition de nombreuses sauces. Cela signifie que ce même mot peut avoir une utilisation concrète liée à la botanique et à la gastronomie, plus une utilisation figurée dans le sens de l'argent comme dans l'exemple suivant : «c'est le gombo qui va leur filer entre les doigts. » (Taalé, P. «Les bouchers font la grève du couteau», Observateur Paalga, 24/11/06). Très souvent utilisé en Afrique noire, façon a surtout une connotation plaisante et assume une fonction d'adverbe qui signifie d'une drôle de façon, d'une manière bizarre ou défectueuse. Gisèle 
Prignitz utilise cet exemple afin d'expliquer que parfois (cet adverbe modulateur nous appartient totalement) noms ou adjectifs se transforment en adverbes et cela parce que "Le vocabulaire à base française subit aussi une "naturalisation" africaine par changement de catégorie grammaticale » (Prignitz 56).

Un cas significatif est celui d'un néologisme composé d'un nom plus un adjectif «nomadisme politique » (Taalé, P. "Sous les déchets toxiques, un deal juteux », Observateur Paalga, 15/09/06), utilisé pour désigner ceux qui «font juste la politique du tube digestif » parce qu'ils changent continuellement de parti politique. Cette expression, présente dans la presse de plusieurs pays africains, est reprise par un journaliste canadien qui en fait le titre d'un de ses articles, «Nomadisme politique au Canada » où le sous-titre explique «On croirait à tort que l'Afrique détenait le monopole de certaines pratiques politiques honteuses. ${ }^{15}$. Ce néologisme a donc été forgé en Afrique où le problème s'est manifesté, mais on y fait recours dans d'autres pays francophones aussi, même si très éloignés. Voilà alors un cas où un terme curieux semble naître et se répandre dans la seule communauté francophone, phénomène qui pourrait marquer le prélude du développement de deux réalités séparées, à savoir le français central et le français périphérique, qui se développent en parallèle.

Une expression particulière concerne le Président ivoirien et sa difficulté à quitter le pouvoir : «[...] la communauté internationale ne peut pas mettre fin au mandat à rallonges du chef de l'Etat ivoirien [...] » (JJ, « Paix et sécurité. L'Afrique soigne ses plaies à l'eau de Cologne », Journal du Jeudi, $\left.\mathrm{n}^{\circ} 788,26 / 10-01 / 11 / 06\right)$. Expression que l'on pourrait utiliser pour la presque totalité des hommes de pouvoir africains qui ne savent pas renoncer au pouvoir. Ainsi, les journalistes s'amusent-il à inventer des pseudonymes à l'égard du Président Blaise Compaoré par rapport au village de ses origines «l'enfant terrible de Ziniaré » (Taalé, P. "L'offre de Gbagbo », Observateur Paalga, 11/09/06) et à sa résidence, symbole du pouvoir, «le locataire du palais Kos-yam » ${ }^{16}$. Ce dernier nom est un terme mooré forgé à partir des termes «Kossé » (demander) et "Yam » (intelligence, sagesse). Il désigne le nouveau Palais présidentiel construit dans le tout nouveau quartier résidentiel et administratif de Ouaga 2000, situé à la périphérie sud de la capitale. La signification du terme est donc « demander la sagesse (pour gouverner) ».

Un autre phénomène souvent présent dans notre corpus se situe à mi-chemin entre lexique et grammaire, $\mathrm{vu}$ qu'il concerne les possibilités de rection des mots dont on modifie les cas de construction. On assiste ainsi à l'utilisation directe du verbe «solliciter". En français central, ce verbe s'accompagne d'un élément abstrait (genre : « solliciter l'attention »), alors qu'ici il est utilisé à la place du verbe " utiliser » et donc avec une signification pragmatique : «Des discours en français, anglais, espagnol, arabe, voilà

15 Voir BASSOLE, André «Nomadisme politique au Canada», L'Express, 07/03/06 publié dans le site: www.lexpress.to/forum/62

${ }^{16}$ Cette dernière expression est très proche de ce qu' on peut lire dans la presse française. 
une rencontre où le casque a été très sollicité » (Kindo, E. "Le clap d'un nouveau partenariat Sud-Sud», Sidwaya, 02/12/2006). Dans le cas de certains verbes, les modifications de rection sont souvent accompagnées par un glissement sémantique, comme dans l'exemple suivant intervenu à l'occasion des funérailles de Ki-Zerbo: "Arrivés à deux kilomètres du domicile du défunt à l'entrée de Toma, les Mossé bloquent la voie. Munis d'arcs et de fléchettes, ils exigent que le "vieux Samo " s'affranchisse d'abord » (Ouedraogo, D. E. «Joseph Ki-Zerbo conduit à sa dernière demeure », Sidwaya, 09/12/2006). En français standard, on dit «s'affranchir de quelque chose » tandis qu'ici on utilise ce verbe à la forme transitive pour dire " payer quelque chose en argent à valeur symbolique pour pouvoir passer». Deux autres verbes sont utilisés dans un contexte similaire : il s'agit de «voir » pour demander de l'argent de manière illicite et de «bouffer » qui désigne quelqu'un qui profite de sa situation pour détourner des sous. Comme l'explique Gisèle Prignitz, c'est surtout au niveau de la connotation qu'on enregistre des changements de relief (Prignitz 56).

Ce que le lecteur occidental remarque immédiatement, ce sont les expressions empruntées à la réalité occidentale et appliquées sans aucune modification à la vie en Afrique, par exemple : «En bons chats échaudés, les Burkinabè craindront le froid de janvier, le temps d'être définitivement rassurés. » (JJ, « 2007. Bon annè ou pas ? », Journal du Jeudi, $\left.\mathrm{n}^{\circ} 798,04-10 / 01 / 07\right)$. Une utilisation qui est exactement copiée et qui peut être utilisée seulement du point de vue métaphorique pour des raisons climatiques bien évidentes. Dans un autre cas, une expression du français standard est réinterprétée en un jeu de mot: "Un couvre feu couve $d u$ feu» (JESP, "Couvre feu, un mot si lointain », Sidwaya, 21/12/2006). C'est ainsi qu'en ajoutant un élément modificateur, l'on obtient l'effet ironique recherché pour retenir l'attention du lecteur.

Les particularités mises en relief par ce corpus d'articles de presse sont à notre avis remarquables, surtout pour la multiplicité et la finalité des variantes. Pour la plupart, il s'agit d'écart instables, mais déjà symptomatiques de la vitalité du français d'Afrique, vitalité encore plus évidente dans ses traits morphosyntaxiques jusqu'à présent moins sujets à une analyse approfondie.

\section{Remarques sur la morphosyntaxe}

Une lecture attentive de ce type de textes prouve que les journalistes burkinabè savent manier la langue française de manière pertinente et élégante. On peut cependant discerner quelques tendances principales du point de vue de la morphologie et de la syntaxe qui sont d'un grand intérêt pour notre analyse car elles s'éloignent de la norme. Cependant, nous éviterons toute justification normative en relation à la grammaire des fautes aussi bien qu'explicative par rapport à l'interférence des langues ethniques (Gandon 70). 
Tous les linguistes qui se sont occupés du français d'Afrique se concentrent sur deux aspects qui ne sont pas difficiles à repérer. La première caractéristique concerne l'omniprésence du déictique - là, typique de l'oralité, mais passé également à l'écrit comme dans la phrase: "En tout état de cause, l'usage de ces pesticides-là, on en reparlera » (Taalé, P. «Suicide ou meurtre à Ouaga 2000 », Observateur Paalga, 09/10/06).

La deuxième observation porte sur une utilisation assez libre des prépositions. Les expressions de lieu sont souvent atténuées comme dans le cas où le journaliste parle de Ouaga $2000:$ « [...] quand il m'arrive de passer vers là-bas et de le regarder avec mes yeux de profane [...]. » (Taalé, P. «Les députés de la Gnagna et le triple assassinat de Pièla », Observateur Paalga, 01/12/06) où la préposition affaiblit toute idée de précision. Tantôt on insiste sur certaines prépositions dont on préfère l'utilisation par rapport à d'autres : «Restons dans le sillage de la grande muette pour dire que pour compter du 03 février 2007, un officier hors du commun s'en ira à la retraite. » (Taalé, P. « Les têtes commencent à tomber dans l'armée », Observateur Paalga, 12/01/07), tantôt on suit la règle principale et on simplifie comme à propos $\mathrm{d}^{\prime}$ un ancien ministre de la Justice : «[...] et cela avant être ministre des Affaires étrangères. " (Taalé, P. "Quand les dieux se rencontrent", Observateur Paalga, 15/12/06). Parfois, on déroge à toute règle comme lorsqu'on lit : « À monsieur Issaka Korogo de savoir mettre davantage d'orthodoxie par la gestion de ses affaires [...]. » (Ibidem), au sens de dans.

Certaines prépositions complexes sont également remarquables. C'est surtout après que qui pose problème dans le choix du subjonctif : "Ce fut ainsi que débuta à 21h, la veillée funèbre après que des autorités religieuses eussent signé le livre des condoléances. » (Ouedraogo, D. E. "Joseph Ki-Zerbo conduit à sa dernière demeure », Sidwaya, 09/12/06), ainsi que «Selon Clotilde Ouédraogo, c'est sa fille aînée de 6 ans qui a reçu en première position, le jour de la distribution, les comprimés après que les agents aient demandé son âge » (Ouedraogo, D. E. "Lutte contre la bilharziose dans la Boucle du Mouhoun », Sidwaya, 13/12/2006). Il s'agit en réalité d'une adaptation linguistique qui lie toujours « que » à un subjonctif correspondant au verbe de la principale.

En ce qui concerne les pronoms démonstratifs, on remarque qu'ils sont souvent suivis d'un adjectif qui les détermine lorsque la construction régulière consisterait à utiliser un article défini (Grevisse 1031). Prenons quelques exemples : "Cette mission fait suite à celle multisectorielle effectuée par le Centre marocain de promotion des exportateurs [...]. » (Taalé, P. «L'offre de Gbagbo », Observateur Paalga, 11/09/06), par rapport aux victimes des émeutes de décembre 2006 : «Quant à celles policières, [...], elles reposent au cimetière de Taabtenga. » (Taalé, P. «La Noël kenyanne de Blaise », Observateur Paalga, 29/12/06) et toujours sur le même sujet: «Les crépitements des armes légères et les détonations de celles lourdes résonnent encore aux oreilles des Ouagalais [...]. » (Taalé, P. «Les têtes commencent à tomber dans l'armée », Observateur Paalga, 12/01/07). Dans ce cas, il s'agit d'une simplification qui ne tient en aucun compte les contraintes grammaticales. 
Maintes perplexités surgissent devant l'utilisation du pronom en pour lequel Grevisse aussi parle de certains cas redondants ${ }^{17}$. Dans nos exemples, on assiste toutefois à une construction syntaxique totalement faussée par une reprise pronominale erronée vu qu'elle engendre une répétition. Considérons la première phrase liée à un pesticide particulièrement dangereux : «[...] notre brave agriculteur avait oublié que ces mêmes céréales pulvérisées de pesticide, lui et sa famille en consommeraient. [...] Séni et un de ses fils en ont succombé » (Taalé, P. «Suicide ou meurtre à Ouaga 2000 », Observateur Paalga, 09/10/06). Dans d'autres cas, nous n'arrivons pas à comprendre pourquoi ce même pronom, qui serait nécessaire, manque totalement. On peut lire à propos de la production de bière : «À cette polémique de Ouagadougou s'ajoute une autre à Bobo, au centre de laquelle il y a, encore, l'argent. » (Taalé, P. «Enlèvement kamikaze sur Babanguida », Observateur Paalga, 02/02/07).

Certaines constructions font défaut dans quelques articles vu qu'on y retrouve des formes absolument désuètes ou erronées en français standard. À deux reprises, nous avons souligné une construction impersonnelle utilisant le pronom personnel « il » plus un verbe au passé composé. La première de ces constructions porte sur l'assassinat du journaliste Zongo : «Et de poursuivre que les confrères (NDLR : journalistes) n'assisteront pas passivement à l'enterrement $\mathrm{du}$ dossier. Il s'est par ailleurs rappelé la présence parmi eux, il y a 2 ans (le 13 décembre 2004) sur les mêmes lieux, du Pr Joseph Ki-Zerbo, décédé le 4 décembre dernier. » (Karama, K. A. "VIIIe anniversaire du drame de Sapouy ", Sidwaya, 14/12/2006). Nous trouvons la seconde, à propos du recensement de la population avant les élections législatives de 2007 : «Pour le moment, c'est l'Etat burkinabè qui en est le principal bailleur de fonds. Mais, il est attendu la contribution des partenaires. » (Emmaüs, J. et Karama, A. K. « Le recensement de la population ne vise aucun but fiscal », Sidwaya, 08/12/2006).

Dans une forme affirmative, c'est le verbe qui pose un problème de concordance : un sujet évidemment singulier est suivi d'un verbe au pluriel parce qu'on l'assimile à d'autres personnages qui accomplissent la même action. C'est le cas de plusieurs descriptions de rendez-vous politiques : «Le mardi 24 octobre 2006, le haut-commissaire du Kadiogo accompagné du Sécrétaire général ont rencontré le maire et le Conseil municipal de Tanghin-Dassouri. » (Taalé, P. «Le poker-menteur de Ménard», Observateur Paalga, 27/10/06).

Parfois, même la forme négative semble déroger à la règle. La négation s'effectue alors de manière inattendue parce qu'incomplète : tantôt un simple adjectif indéfini négatif semble suffire, «[...] il s'est inquiété de la validité des audiences foraines qui se dérouleront sous aucune autorité préfectorale » (R.K., "Audiences foraines à Massala », Le

\footnotetext{
${ }^{17}$ Grevisse dit cela à propos de $y$ et en lorsqu'ils reprennent devant le verbe des compléments détachés au début ou en fin de phrase (Grevisse 1000).
} 
Matin d'Abidjan, 05/08/2006), tantôt la négation de deux infinitifs ne porte que sur le premier, le deuxième étant introduit par une coordonnée à valeur positive et non par ni: « S'agissant du Premier Ministre, il n'a pas voulu revoir et prendre en main les Forces dites de Défense et de Sécurité » (D.B., «Situation socio-politique : les erreurs que Banny et le RHDP doivent corriger ", Le nouveau Réveil, 02/08/2006.). Une certaine insécurité se manifeste à l'égard de la négation de l'infinitif « [...] même si l'Etat semble n'avoir pas dit son dernier mot.» (Taalé, P. «Quand les dieux se rencontrent», Observateur Paalga, 15/12/06) ainsi qu'un manque de précision concernant les adverbes à propos d'un certificat pour un étudiant: "Là-bas aussi on ne vend pas de ce timbre. » (Taalé, P. "Les têtes commencent à tomber dans l'armée », Observateur Paalga, 12/01/07).

C'est certainement à cause de la vitesse des informations et de la nécessité de leur publication que l'on trouve des exemples de transcriptions du discours direct à la forme indirecte. Cela ressort de toute évidence du pronom personnel qui suit : "ZONGO et LANKOANDE ont affirmé que 56 veuves de l'entreprise ont pleuré devant nous. » (Ouedraogo, H. "Réaction de Me Halidou Ouédraogo à deux ex-travailleurs de OK ", Sidwaya, 01/12/2006) ou de cette forme interrogative directe : " Il ne savait pas quand est-ce qu'il a été embauché, quand est-ce qu'il a été licencié. » (Ibidem).

La construction se fait lourde et enchevêtrée lorsqu'une phrase se compose de bien des relatives comme dans l'exemple qui suit : "Chaque fois, on aura constaté que le mode même de pensée était en cause, qui fait du pouvoir, de la souveraineté et de l'intégration des notions antinomiques » (JJ, «Silence, on intègre!», Journal du Jeudi, $\mathrm{n}^{\circ}$ 801, 2531/01/07). Ici, la deuxième relative est liée à la première d'une manière incorrecte comme subordonnée d'une subordonnée. Parfois, le problème semble lié à une surabondance de détails comme il arrive dans la phrase : "Pour des pays situés dans le même espace, c'en est un peu trop, ce qui ne facilite guère les échanges » (Ibidem) à propos des différentes monnaies existant en Afrique sub-saharienne : le F CFA des pays francophones, le cédi ghanéen, le naïra nigérian et le dalasi gambien.

Dans la réalité des faits, c'est la surabondance de propositions qui se révèle assez problématique lorsqu'il s'agit de formes surchargées, ou bien qui réunissent trop de subordonnées et de prépositions. En voilà un exemple: «En somme, en plus de la performance de la machine électorale qui a fait ses preuves, il faudra lui ajouter ce supplément de popularité vraie et non mécanique pour se faire une assise moins sujette à angoisses pour les animateurs dans l'échafaudage quasi quotidien de parades pour demeurer leader envers et contre tous. » (JJ, «CDP. Le congrès d'un grand parti n'est pas petit », Journal du Jeudi, $\mathrm{n}^{\circ}$ 790, 09-15/11/06). La coordination semble donc manifester certaines particularités assez récurrentes, même si quelques exemples relevés dans la presse africaine ne semblent pas radicalement différents de ceux qui émaillent la presse française. Partout en effet, les journalistes - pressés par les événements et les échéances ont tendance à simplifier pour retrouver ensuite le tour synthétique en vertu de la loi du 
moindre effort. Voilà ce qu'on trouve à l'occasion de la recherche d'un certificat médical pour un étudiant: "En effet, comment a-t-on idée d'arrêter la vente d'un timbre sous prétexte qu'on en attend un nouveau, qui n'est pas encore là, et sans en prévenir les usagers, ne serait-ce que par un petit communiqué ?» (Taalé, P. «Les têtes commencent à tomber dans l'armée », Observateur Paalga, 12/01/07).

\section{En guise de conclusion}

S'il est vrai que les variantes lexicales sont plus évidentes et peut-être plus amusantes aussi, on ne peut pas mettre au second plan les variantes d'ordre morphosyntaxique : disséminées de manière assez régulière, elles sont à surveiller parce qu'elles sont destinées petit à petit à s'incruster. D'autres caractéristiques s'imposent qui ne peuvent rentrer dans les sections évoquées ci-dessus. Tout au long de la lecture de ces articles de presse, nous avons pu remarquer des fautes de frappe que l'on peut aisément trouver même dans la presse de l'Hexagone. Il s'agit de coquilles déterminées par la rapidité de la composition et l'urgence des différentes situations. Par exemple, la répétition et l'homophone qui suivent : «Les résultats ont été portés à la connaissance de la Presse au cour d'une conférence de presse. » (Ouedraogo, H. « Réaction de Me Halidou Ouédraogo à deux ex-travailleurs de OK », Sidwaya, 01/12/2006), et encore : "Ces derrières années, la diplomatie burkinabè a remporté des succès. » (Karama, K. A. "Ce qu'attend Blaise Compaoré de ses représentants à l'étranger», Sidwaya, 20/12/2006). On assiste également à l'utilisation erronée de certains termes moins usités tels que : "C'était la débande. » (Kere, A. "Heurts entre policiers et militaires à Ouagadougou », Sidwaya, 21/12/2006) Des cas-limite qui occupent également certains titres : «Bon annè ou pas ? (JJ, 2007. «Bon annè ou pas ? », Journal du Jeudi, n 798, 04-10/01/07) ou qui se font remarquer pour une volonté d'exagération : «Celui-ci depuis son installation n'a pas fonctionné faute de manque de moyens. » (Ouedraogo, D. E. Sanga, B. Hema, C. « Lutte contre la traite et l'exode d'enfants. Réprimer pour donner l'exemple », Sidwaya, 01/12/2006).

D’un autre côté, Pascal Somé remarque un souci d'hyper-correctisme à l'oral, ce que nous avons également trouvé à l'écrit surtout dans deux situations bien précises (Somé 2006 : 117). La première se vérifie lorsque le journaliste choisit de retranscrire certains documents en en soulignant les fautes et en donnant immédiatement après la correction aux lecteurs. C'est le cas par exemple à l'occasion d'une distribution massive de médicaments spécifiques, un épisode d'extrême importance touchant la santé de la population. La fiche du docteur responsable du district se termine par «Je vous l'envoie avis et une meilleur prise en charge » (NDLR : la faute d'orthographe du mot «meilleur» est du document) signé «traitant» avec le tampon « régiment parachutiste commando » (D. E. Ouedraogo, "Lutte contre la bilharziose dans la Boucle du Mouhoun», Sidwaya, 13/12/2006) et un peu plus loin " pour vomissement bilieux accompagnée (NDLR : la faute d'orthographe - e muet a accompagnée provient du document) de diarrhée liquidienne le tout évoluant depuis 96 heures environ (NDLR : 4 jours environ après la prise) » (Ibidem). 
Le second cas que nous avons remarqué concerne l'explicitation des chiffres toujours accompagnés par l'indication en lettres. En voici quelques exemples : «Depuis un certain temps $\operatorname{deux}(02)$ personnes $[. .] »$, « [...] et pour cela nous avons pris trois (03) jours [...]», « Nous avons examiné quatre (04) dossiers [...]» (Ouedraogo, H. "Réaction de Me Halidou Ouédraogo à deux ex-travailleurs de OK », Sidwaya, 01/12/2006). S'agissant de cet usage, Gandon se demande s'il s'agit d'une ancienne influence de l'administration coloniale, tout en considérant qu'en France cela est réservé aux données administratives, juridiques et financières (Gandon 84).

Les comportements que nous venons d'évoquer sont symptomatiques des tentatives d'appropriation de la langue française à travers de multiples processus - de vernacularisation ou de véhicularisation au contact des langues nationales. À partir d'une certaine vision du monde africain, ainsi que de modes de conceptualisation spécifiques, nous sommes arrivés à déchiffrer des tendances inattendues par rapport au «français central », mais notre analyse ne peut avoir aucun caractère d'exhaustivité. Tous ces phénomènes ne sont que la manifestation d'une réalité in fieri.

Il est sûr que pour arriver à redéterminer l'usage d'une langue, il faut bien la maîtriser, d'où les jeux de mots, les néologismes et toutes ces expressions créées sur la base de l'urgence d'une actualité en éternel mouvement. Le mélange seul constitue la normalité en milieu communicatif, une normalité qui se veut protéiforme et enrichissante. Le plurilinguisme n'est pas une entité abstraite, mais un devenir palpable, une forme de création qui pose les jalons d'un chemin dont on ne connaît pas encore le point d'arrivée.

Le français des journaux se confirme comme un excellent support pour l'étude descriptive d'une norme d'usage, celle des plus lettrés, mais aussi comme modèle susceptible de servir de norme de prestige, et de prendre le relais de la norme scolaire, pour une partie intermédiaire de la population. Cela signifie que le sentiment d'appropriation de la langue française au Burkina est complet et prouvé par la vitalité des tendances analysées. Bien d'autres restent à sonder, parce qu'il est désormais prouvé que « le français s'africanise » (Somé 117). Les possibilités à exploiter sont infinies, on ne peut évaluer si toutes seront durables, mais les effets de surprise sont certainement garantis par cette variabilité dynamique qui fait de l'écriture une activité d'expérimentation sous « tous les soleils ».

Il reste cependant prouvé que l'originalité de cette production journalistique réside non pas au niveau des contenus, mais plutôt de la langue (voire des langues) qui confère (confèrent) une certaine particularité linguistique aux multiples articles. C'est ainsi qu'à la langue française standard et neutre de la presse de l'Hexagone, s'en substitue une autre d'expression décidément plus burkinabè, une production aux caractéristiques nationales dont on trace les pistes à parcourir et qui incarne les réalités de passage. 


\section{Bibliographie}

- Bavoux C., L.-F. Prudent et S. Wharton. Normes endogènes et plurilinguisme. Aires francophones, aires créoles. Lyon : ENS Editions, 2008.

- Calvet, L.-J., Griolet, P. (sous la direction de). Impérialismes linguistiques hier et aujourd'hui. Aix-en-Provence : Inalco-Edisud, 2005.

- Calvet, Louis-Jean. La Guerre des langues et les politiques linguistiques. Paris: Hachette, 1999.

- Da, Evariste. “Au carrefour de la palabre.” Notre Librairie 101 (1990) : 6-9.

- Dion R., Lüsebrink H.-J. et Riesz J. Ecrire en langue étrangère. Interférences de langues et de cultures dans le monde francophone. Québec: Editions Nota bene, 2002.

- Dumont, Pierre. L'Interculturel dans l'espace francophone. Paris : L'Harmattan, 2001.

- Eloy, J.-M. (sous la direction de). La Qualité de la langue? Le cas du français. Paris: Champion, 1995.

- Gadet, Françoise. Le Français ordinaire. Paris : Colin, 1989.

- Gandon, F.-M. "Appropriation et syntaxe du français écrit dans la presse de Ouagadougou (Burkina Faso): préposition, rection, pronoms." Langue Française 104 (1994) : 70-88.

- Grevisse, M. Le bon usage. Paris : Duculot, 2001.

- Grossmann, François, Paveau, Marie-Anne, Petit, Gérard (éds.). Didactique du lexique: langue, cognition, discours. Grenoble : ELLUG, 2005.

- Gueunier, Nicole, Genouvrier, Emile, Khomsi, Abdelhamid. Les Français devant la norme. Paris : Champion, 1978.

- Hagège, Claude. L'Homme de paroles. Contribution linguistique aux sciences humaines. Paris : Fayard, 1985.

- Hagège, Claude. Combat pour le français. Paris : Odile Jacob, 2006.

- Huannou, Adrien. Francophonie littéraire et identités culturelles, Actes du Colloque du Grelef (Cotonou, 18-20 mars 1998). Paris : L'Harmattan, 2000.

- Keita, Alou. "Fantaisie lexicale et néologie : le cas de JJ : presse écrite en français au Burkina Faso." Le français en Afrique 12 (1998). 20 Juin 2008. <http://www.unice.fr/ILFCNRS/ofcaf/12/Keita.htm>.

- Laroussi, Foued, Babault, Sophie (sous la direction de). Variations et dynamisme du français. Une approche polynomique de l'espace francophone. Paris : L'Harmattan, 2001.

- Manessy, Gabriel. Le Français en Afrique noire. Mythe, stratégies, pratiques. Paris : Éditions L'Harmattan, 1994.

- Nikiema Norbert. “La situation linguistique.” Notre Librairie 101 (1990) : 16-19.

Porcher L.-V. Faro-Hanoun. Politiques linguistiques. Paris : L'Harmattan, 2000.

- Prignitz, Gisèle. "Rôle de l'argot dans la variation et l'appropriation : le cas du français au Burkina Faso" Langue française 104 (1994) : 49-63.

- Prignitz, Gisèle. "La mise en scène du plurilinguisme dans l’œuvre de Jean-Hubert Bazié. Une représentation sociolinguistique du Burkina Faso." Etudes africaines 163-164 (2001). 14 Juillet 2008. <http://etudesafricaines.revues.org>. 
- Robillard, Didier (de), Beniamino, Michel (sous la direction de). Le Français dans l'espace francophone. Description linguistique et sociolinguistique de la francophonie, tome I et II. Paris : Champion, 1993 et 1996.

- Sissao, Alain Joseph. “La question du métissage dans l'écriture du roman burkinabè contemporain." Etudes africaines 163-164 (2001). $14 \quad$ Juillet 2008. $<$ http://etudesafricaines.revues.org $>$.

- Somé, Pascal. "Le plurilinguisme en héritage." Le français dans le monde, numéro spécial (2006) : 108-122.

\section{Sitographie}

- Etudes africaines. 1980. 30 Mai 2008. <http://etudesafricaines.revues.org>.

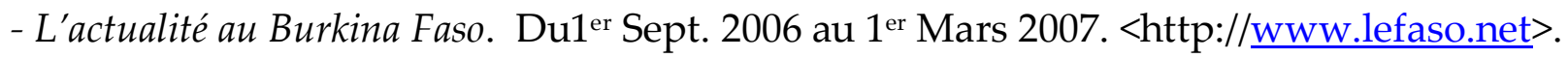

- Trésor de la langue française au Québec. 25 Avril 2008.

$<$ http://www.tlfq.ulaval.ca/axl/afrique/burkina.htm>.

- Le français en Afrique. 14 Juillet 2008. <http://www.unice.fr/ILF-CNRS/ofcaf/6>. 